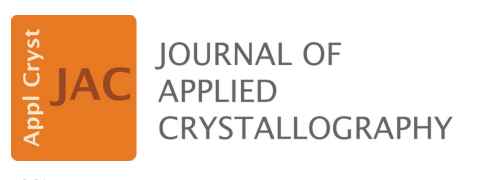

ISSN 1600-5767

Received 25 July 2016

Accepted 12 November 2016

Edited by K. Chapman, Argonne National Laboratory, USA

Keywords: high pressure; time of flight; diamond-anvil cells; attenuation.

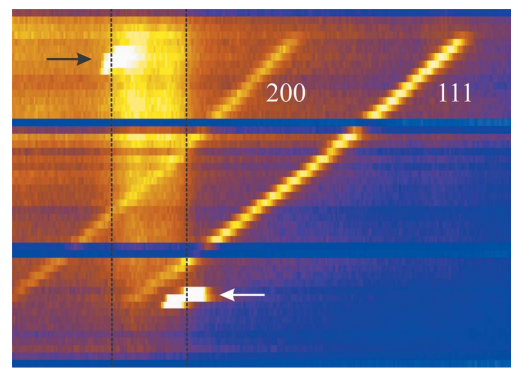

C 2017 International Union of Crystallography

\section{Radiation attenuation by single-crystal diamond windows}

\author{
M. Guthrie, ${ }^{a *}$ C. G. Pruteanu, ${ }^{b}$ M.-E. Donnelly, ${ }^{b}$ J. J. Molaison, ${ }^{c}$ A. M. dos Santos, ${ }^{c}$ \\ J. S. Loveday, ${ }^{b}$ R. Boehler ${ }^{c, d}$ and C. A. Tulk ${ }^{c}$
}

${ }^{\mathbf{a}}$ European Spallation Source, ERIC, Lund, Sweden, ${ }^{\mathbf{b}}$ SUPA, School of Physics and Astronomy and Centre for Science at
Extreme Conditions, University of Edinburgh, Edinburgh, UK, ${ }^{\mathrm{C}}$ Oak Ridge National Laboratory, Oak Ridge, TN, USA, and
${ }^{\mathbf{d}}$ Carnegie Institution of Washington, Washington, DC, USA. ${ }^{*}$ Correspondence e-mail: malcolm.guthrie@esss.se

As artificial diamond becomes more cost effective it is likely to see increasing use as a window for sample environment equipment used in diffraction experiments. Such windows are particularly useful as they exhibit exceptional mechanical properties in addition to being highly transparent to both X-ray and neutron radiation. A key application is in high-pressure studies, where diamond anvil cells (DACs) are used to access extreme sample conditions. However, despite their utility, an important consideration when using single-crystal diamond windows is their interaction with the incident beam. In particular, the Bragg condition will be satisfied for specific angles and wavelengths, leading to the appearance of diamond Bragg spots on the diffraction detectors but also, unavoidably, to loss of transmitted intensity of the beam that interacts with the sample. This effect can be particularly significant for energy-dispersive measurements, for example, in time-of-flight neutron diffraction work using DACs. This article presents a semi-empirical approach that can be used to correct for this effect, which is a prerequisite for the accurate determination of diffraction intensities.

\section{Introduction}

When used as a window for scattering experiments, the most important attributes of diamond are its simple crystal structure - space group $(F d \overline{3} m), a=3.5665 \AA$ (Sato et al., 2002) and a relatively low mass absorption for both X-ray and neutron radiation. For diamond anvil cells (DACs), which are commonly used with a transmission geometry, the incident beam must traverse an appreciable thickness of diamond (typically $1-3 \mathrm{~mm}$ ) prior to reaching the sample, making diamond attenuation an important consideration. The effect of this transit on the intensity of the beam is twofold: (i) mass absorption, where intensity is lost as it is absorbed by individual atoms in the material, and (ii) intensity losses due to Bragg reflections from the diamond lattice. While the former effect can be substantial at lower X-ray energies (e.g. $\mathrm{Cu} \mathrm{K \alpha})$, it is relatively simple to apply a geometric correction (Angel, $2004)$ and, for hard X-rays ( $>30 \mathrm{keV})$ and especially neutrons, the effect can be essentially neglected. However, the latter effect, that of Bragg losses, has a much more complicated dependence on both scattering vector $(\mathbf{Q})$ and diamond microstructure and must be carefully considered (Loveday et al., 1990). In addition, as observed by Loveday et al. (1990), the magnitude of the losses is strongly strain dependent. In the case of a DAC, where the diamond strain is substantial and varies as a function of sample pressure, the formulation of a correction is especially challenging. 
In our own work, we have used large-volume neutron DACs in energy-dispersive neutron diffraction experiments at the Spallation Neutron Source, Oak Ridge, TN, USA, employing a transmission geometry (Guthrie, Boehler, Tulk et al., 2013). We have conducted transmission measurements of the beam that passes through the DAC, which show clear dips in intensity due to Bragg losses in the polychromatic neutron beam. In order to examine the effect of these dips on sample diffraction intensities, we have conducted these transmission measurements as a function of sample pressure. The effect was indeed found to be pressure dependent and sufficient to modify the relative intensities of Bragg peaks at our highest pressure $(40.4 \mathrm{GPa}$ ) by up to $\sim 15 \%$. Here, we present our study and a semi-empirical approach we have developed to apply a correction for this diamond attenuation effect on the sample Bragg intensities.

\section{Theoretical background}

The Ewald construction provides a way both to visualize which reflections will satisfy the Bragg condition and to determine the wavevectors of the corresponding diffracted beam. The construction is made from two components. The first is the surface that is mapped out by all possible scattering vectors, $\mathbf{Q}=\mathbf{k}_{\mathrm{f}}-\mathbf{k}_{\mathrm{i}}$, where $\mathbf{k}_{\mathrm{i}}$ and $\mathbf{k}_{\mathrm{f}}$ are the incident and final wavevectors, respectively. The vectors $\mathbf{k}_{\mathrm{i}}$ and $\mathbf{k}_{\mathrm{f}}$ are defined in the usual way to be parallel to the incident and diffracted beams, respectively, and to have magnitude $2 \pi / \lambda$ where $\lambda$ is the wavelength of the radiation. For an elastic experiment, the incident and final wavelengths are equal, so the locus of all
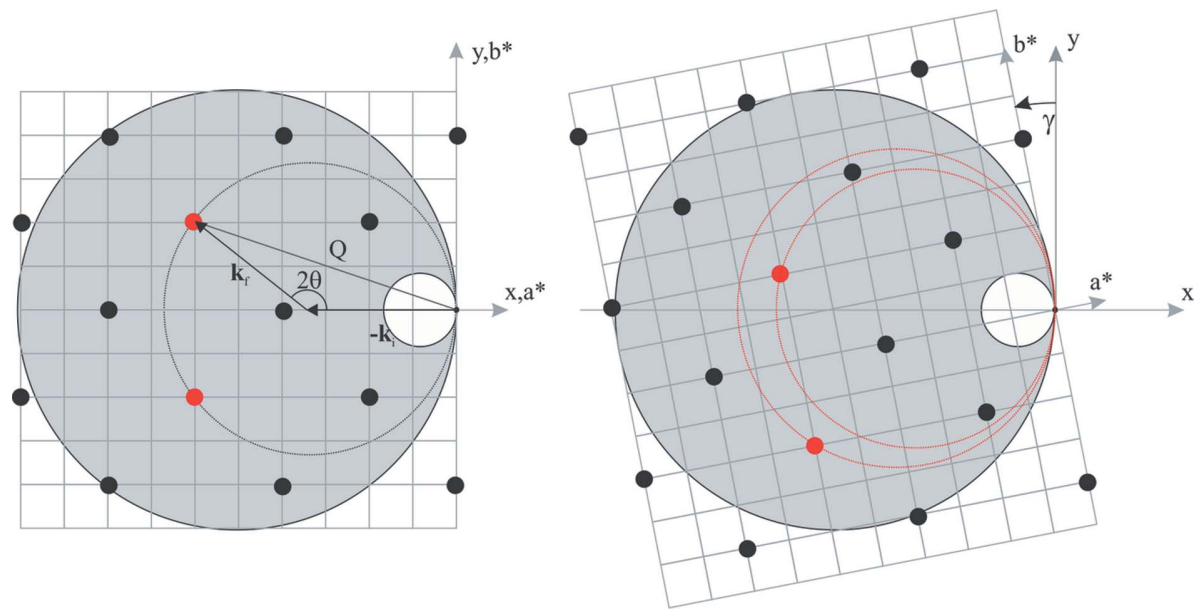

Figure 1

A horizontal slice through reciprocal space showing the Ewald construction. Here, a right-handed set of Cartesian axes is defined by unit vectors $\mathbf{x}, \mathbf{y}$ and $\mathbf{z}$, which together constitute a basis set $\mathbf{X}$ whose origin is at the sample position and which is fixed relative to the neutron beam. The vector $\mathbf{x}$ is defined to be parallel to the incident beam while $\mathbf{z}$ is vertical (pointing out of the page). The grey area represents the volume delimited by the minimum and maximum Ewald radii and is static with respect to $\mathbf{X}$. In the left-hand image, the reciprocal lattice for diamond is shown as solid circles for the case when the [100] axis $\left(\mathbf{a}^{*}\right)$ is parallel to the beam, so that $\mathbf{a}^{*} \| \mathbf{x}$; also, $\mathbf{b}^{*} \| \mathbf{y}$ and $\mathbf{c}^{*} \| \mathbf{z}$. In this orientation, the two lattice points $(\overline{6}, 2,0)$ and $(\overline{6}, \overline{2}, 0)$ (solid red circles) will both satisfy the Bragg condition at the same wavelength, given by the radius of the Ewald sphere that passes through them. In the right-hand image, the crystal (and its reciprocal lattice) is rotated by an angle $\gamma$ about $\mathbf{z}$. In this orientation, $(\overline{6}, 2,0)$ and $(\overline{6}, \overline{2}, 0)$ are intercepted by two different Ewald spheres of different radii, and these reflections will scatter at different wavelengths. possible $\mathbf{Q}$ vectors maps out the surface of a sphere with a radius of $\left|\mathbf{k}_{\mathrm{i}}\right|=\left|\mathbf{k}_{\mathrm{f}}\right|=2 \pi / \lambda$ called the Ewald sphere. The second component of the Ewald construction is the reciprocal lattice of the material of interest. The reciprocal lattice vectors $\mathbf{a}^{*}, \mathbf{b}^{*}$ and $\mathbf{c}^{*}$ \{determined from the crystal lattice in the normal way e.g. $\mathbf{a}^{*}=2 \pi(\mathbf{b} \times \mathbf{c}) /[\mathbf{a} \cdot(\mathbf{b} \times \mathbf{c})]$ etc. $\}$ define a basis set

$$
\mathbf{A}^{*}=\left(\begin{array}{l}
\mathbf{a}^{*} \\
\mathbf{b}^{*} \\
\mathbf{c}^{*}
\end{array}\right)
$$

for the reciprocal lattice. A general reciprocal lattice point is then described by the vector $\mathbf{G}_{h, k, l}$ which is given by

$$
\mathbf{G}_{h, k, l}=h \mathbf{a}^{*}+k \mathbf{b}^{*}+l \mathbf{c}^{*},
$$

where $h, k$ and $l$ are the conventional Miller indices (Ashcroft $\&$ Mermin, 1976). Following Giacovazzo (1998), this can be written in matrix form as

$$
\mathbf{G}_{h, k, l}=\left(\mathbf{a}^{*}, \mathbf{b}^{*}, \mathbf{c}^{*}\right)\left(\begin{array}{l}
h \\
k \\
l
\end{array}\right)=\mathbf{A}^{*} \mathbf{h},
$$

where $\mathbf{h}=(h, k, l)$ is the coordinate matrix.

It can be shown (Ashcroft \& Mermin, 1976) that the Bragg condition for the lattice plane with Miller indices $h, k$ and $l$ is lattice vector, i.e. $\mathbf{Q}=\mathbf{G}_{h, k l}$. If the Ewald sphere is plotted on the reciprocal lattice such that $\mathbf{k}_{\mathrm{i}}$ has the correct orientation then this will be the case when a reciprocal lattice point intersects the Ewald sphere.

For time-of-flight diffraction, a polychromatic incident beam is used with the wavelength of an arriving neutron determined by measuring its transit time from moderator to detector. In this case, it is necessary to consider a continuum of Ewald spheres that together fill a volume of reciprocal space bounded by limiting spheres corresponding to the shortest and longest wavelength used (see Fig. 1). Correspondingly, each of the $N$ reciprocal lattice points in this entire 'Ewald volume' will satisfy the Bragg condition. For each reflection $n=$ $1, \ldots, N$, the magnitude of its scattering vector $\mathbf{Q}_{n}$ and, therefore, the corresponding $d$ spacing is invariant with respect to the orientation of the crystal. However, as the crystal rotates, the direction of $\mathbf{Q}_{n}$ changes and the wavelength of the corresponding Bragg event, $\lambda_{n}$, also changes (Fig. 1).

The information describing the orientation of the reciprocal lattice, with respect to the instrument frame $\mathbf{X}$ (defined in the caption to Fig. 1), and therefore the incident beam, is contained in the $3 \times 3$ orientation 
matrix UB. For a crystal system where the lattice parameters are known, a measurement of the scattering vectors for any two non-colinear reflections is sufficient to constrain the UB matrix. In any diffractometer, the same detectors that measure the sample diffraction signal also inevitably measure the diamond single-crystal reflections, so it is generally possible to obtain an orientation matrix for the diamond lattice. We used the ISAW neutron crystallography software package (Chatterjee et al., 2002) to determine UB. When the UB matrix thus obtained operates on the coordinate matrix $\mathbf{h}$, it returns the coordinates of the corresponding scattering vector $\mathbf{Q}$ in a frame coincident with $\mathbf{X}$ with units of $\AA^{-1}$ :

$$
\mathbf{U B} \mathbf{h}_{n}=\left(\begin{array}{c}
Q_{x} \\
Q_{y} \\
Q_{z}
\end{array}\right)_{n}=\mathbf{Q}_{n} .
$$

Given UB, it is then straightforward to calculate the wavelength $\lambda_{n}$ at which reflection $n$ will result in a loss in transmitted intensity.

\section{Experimental setup}

Our measurements were conducted on the dedicated highpressure diffractometer SNAP on Beamline 3 of the Spallation Neutron Source. The geometry of SNAP has been described in detail previously (Guthrie, Boehler, Molaison et al., 2013). Here we briefly summarize the basic setup. The neutron beam emanating from the instrument flight tube is focused by a $2 \mathrm{~m}$ parabolic guide before hitting the sample, which is $15 \mathrm{~m}$ from the moderator. The final beam size is determined by a small pinhole, formed from hexagonal $\mathrm{BN}$, that is placed $\sim 5 \mathrm{~mm}$ upstream of the sample position. The main diffraction detectors are two square, pixelated, Anger cameras (Richards et al., 2010), which are positioned to the left- and right-hand sides of the sample, in a horizontal plane, both centred on a scattering angle $2 \theta$ of $90^{\circ}$. In our measurements, the DACs are normally aligned with their load axis parallel to the beam and have a seat assembly that limits scattering angles to around $90 \pm 20^{\circ}$ (see Fig. 7 below). In this orientation, the a axes of both diamond lattices are close to parallel to the incident beam direction, although we find that a variation of up to $\sim 2^{\circ}$ is possible, depending on details of culet polishing. At present, the amount by which the plane containing the $\mathbf{b}$ and $\mathbf{c}$ axes of the diamonds is rotated about the beam is not controlled and varies randomly with each loading. Transmitted neutrons are measured with an ORDELA ${ }^{3} \mathrm{He}$ gas monitor, which has an efficiency of $5 \times 10^{-3}$ and an active area of $64 \times 50 \mathrm{~mm}$ and which is located $\sim 650 \mathrm{~mm}$ downstream of the sample.

In order to examine the pressure dependence of the transmission spectra and to test the correction described below (in §4), a sample of Ni powder was measured at a series of increasing pressures. The loading was prepared with $1.5 \mathrm{~mm}$ diamond culets, and with T301 steel gaskets having an initial thickness of $400 \mu \mathrm{m}$, an indent thickness of $\sim 150 \mu \mathrm{m}$ and an inner diameter of $\sim 600 \mu \mathrm{m}$. A new cell design with an integrated-membrane force driver was used. A collimator mounted independently from the cell, and held at a distance of around $5 \mathrm{~mm}$ from the sample, defined a final beam size just smaller than the sample itself (no gasket scattering was evident in the diffraction patterns). In addition to the sample data collections, measurements of a $2 \mathrm{~mm}$-diameter $\mathrm{V}$ rod and its background were collected to normalize the data to the baseline incident flux profile and detector efficiency.

\section{The transmission spectrum and details of the model}

The beam monitor measures the integrated counts of the main beam at a location $\sim 650 \mathrm{~mm}$ downstream of the sample as a function of neutron time of flight (TOF). As described below, the TOF is proportional to the neutron wavelength. As such, all of the many factors affecting the wavelength dependence of the beam (such as moderator temperature, attenuation due to upstream vacuum windows and the efficiency of the focusing guide) multiply with the intrinsic detector efficiency to give the measured counts. However, if we take the ratio of the monitor spectra for a run when the cell is present and a background run where the cell is removed, all of these factors cancel, leaving only the attenuation due to the diamonds and the sample. An example of the resulting spectrum, observed at a low hydraulic load, is shown in Fig. 2.

This pattern shows the characteristic features of the diamond attenuation. These are strong losses that appear at the particular times of flight (or, equivalently, wavelengths) corresponding to Bragg scattering from both diamond anvils. Meanwhile, outside these 'dips' in intensity, the transmission is essentially $100 \%$, which is expected as the mass absorption of carbon for neutron radiation is essentially zero (Sears, 1992). It is evident that such pronounced dips in incident beam intensity must also lower the intensity of sample Bragg reflections measured at those specific wavelengths. As these intensities contain much of the crystallographic information in

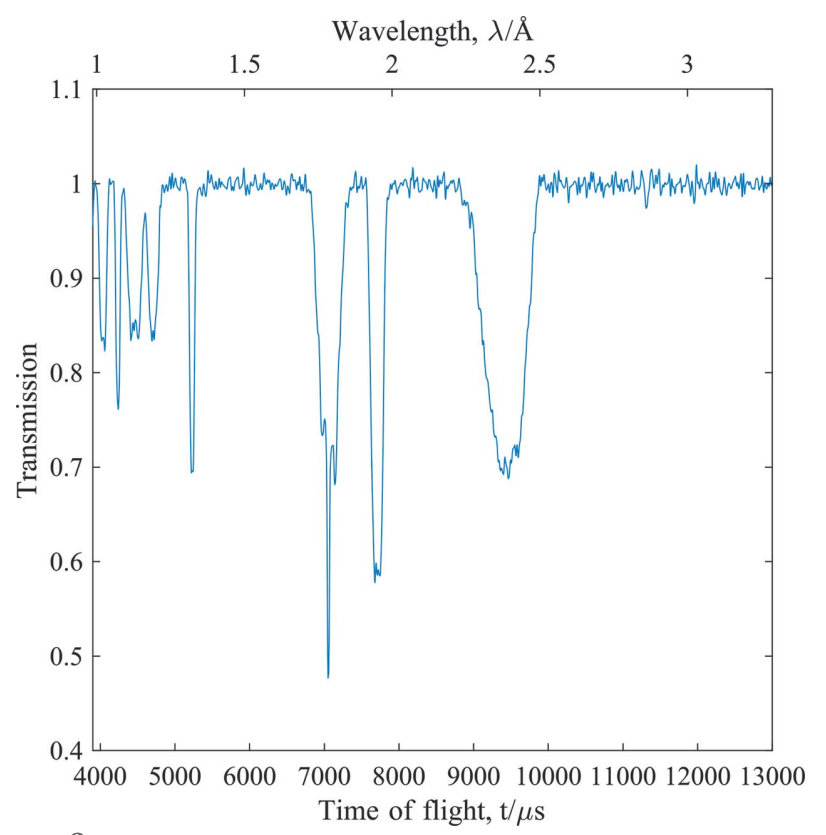

Figure 2

Transmission spectrum through the DAC (and the Ni sample) at a load of 0.5 tonnes. 
a diffraction experiment, it is clearly important to apply a correction for this effect. Previous measurements (Guthrie, Boehler, Molaison et al., 2013) used a vanadium sample measured inside the cell to provide a correction. However, this approach assumed that the effect is pressure invariant while, as reported previously (Loveday et al., 1990) and as shown below, the effect increases with anvil strain.

In principle, the measured pattern in Fig. 2 contains all the required information to calculate such a correction. However, there is a complication in that the transmission data are unavoidably a composite of both diamonds (one upstream and one downstream of the sample) as the beam must pass through both on its route to the transmission monitor. Meanwhile, it is only the attenuation due to the upstream diamond that will affect the beam that interacts with the sample. Therefore, it was necessary to develop a procedure to deconvolve the measured transmission patterns into separate spectra for each individual diamond anvil. The approach adopted was to calculate a simulated dip pattern using a model with variable parameters that could be optimized using a least-squares approach to give the best fit to the measured transmission data. From this model, the individual contributions of the upstream and downstream diamonds can then be extracted. The list of model parameters includes the intensity (or depth) of each dip, making the method analogous to a Le Bail-type profile refinement (Le Bail, 2005) where independent information can be extracted from separate phases contributing to the total measured diffraction pattern.

In order to describe the measured transmission pattern, we developed a parameterization based on a simple summation of Gaussians on a constant background of 1 . While this functional form has no physical meaning, it has the advantage of being simple to calculate and, as shown later, gives a good fit to the measured pattern. The simulated pattern is generated in an iterative loop that - for each diamond, which we label $j=1$ or 2 - runs over all of the $N$ reflections allowed by the diamond space group $(F d \overline{3} m)$ that lie within the Ewald volume (typically $N \simeq 70$ for each diamond). The Gaussian that models the dip due to reflection $n$ is described by three parameters: position $\left(\lambda_{n}\right)$, width $\left(\sigma_{n}\right)$ and area $\left(\kappa_{n}\right)$. Finally, all of the Gaussians for diamond $j$ are scaled by an overall scale factor $\Gamma_{j}$ (this parameter is useful when both diamonds are constrained to have dip intensities as described below). The corresponding model for the transmission spectrum for diamond $j$ with orientation matrix $\mathbf{U} \mathbf{B}_{j}, T_{j}^{\text {calc }} \equiv T_{j}^{\text {calc }}(\lambda)$, is given by

$$
T_{j}^{\text {calc }}(\lambda)=1-\Gamma_{j} \sum_{n=1}^{N} \kappa_{n} \exp \left[-\frac{\left(\lambda-\lambda_{n}\right)^{2}}{2 \sigma_{n}^{2}}\right],
$$

which is calculated at each of the $K$ discrete wavelength values $\lambda_{K}$ between the maximum and minimum wavelength of the experimental measurement.

As is evident from equation (5), the model for the transmission spectrum depends on a set of parametric variables, which are determined as follows. The $\lambda_{n}$ for each of the $n$ reflections satisfying the Bragg condition for diamond $j$ can be calculated using the orientation matrix $\mathbf{U} \mathbf{B}_{j}$. However, in initial testing, it was observed that imprecision in the orientation matrix determination (due to both a finite pixel size of $\sim 0.5^{\circ}$ and relatively low sampling angle for reflections: $70<$ $2 \theta<100^{\circ}$ ) was $\sim 1^{\circ}$. Therefore, to take account of this, three 'adjustment' angles were introduced as additional variables in the fit. These angles, labelled $\alpha, \beta$ and $\gamma$, correspond to successive rotations of all $\mathbf{Q}_{n}$ about $\mathbf{x}, \mathbf{y}$ and $\mathbf{z}$ (as defined in Fig. 1, which illustrates a rotation of $0,0, \gamma)$ and, therefore, modify the dip wavelengths $\lambda_{n}$. In addition to these adjustment angles, it was also necessary to consider the effect of the load on the diamond lattice, which can compress measurably. In order to take account of this in the model, a multiplicative scale factor $\delta$ is introduced to correct the wavelength of a dip, such that $\lambda_{n} \rightarrow \delta \lambda_{n}$ (with $\delta \leq 1$ when the diamonds are under load).

The width of a given dip, $\sigma_{n}$, was taken to have a parabolic dependence on $\lambda$, such that $\sigma_{n}=\varepsilon_{1} \lambda_{n}+\varepsilon_{2} \lambda_{n}^{2}$, requiring only two parameters $\left(\varepsilon_{1}\right.$ and $\left.\varepsilon_{2}\right)$ to describe the width of all $\sim 140$ dips from both diamonds. At the beginning of refinement, the area of each $\operatorname{dip} \kappa_{n}$ is given an initial value equal to the calculated structure factor squared for the relevant reflection $n$, determined using a GSAS (Larson \& Von Dreele, 1994) simulation. During subsequent fitting, the values of $\kappa_{n}$ are free parameters that can be varied to fit the measured spectrum. However, they are constrained to be identical for equivalent reflections.

It is possible to constrain the series of $\kappa_{n}$ to be the same for both diamonds so only the parameters $\Gamma_{j}$ differ between the two diamonds. This reduces the total number of parameters by $\sim 50 \%$ and is a reasonable approximation: allowing for slight differences in diamond thickness (and therefore total attenuation) but assuming both diamonds have similar microstructure (e.g. mosaic spread and strain). Alternatively, $\kappa_{n}$ can optionally be completely unconstrained for each diamond, in which case the scale factors $\Gamma_{j}$ are set equal to one and ignored.

The summations in equation (5) run across the $N$ coordinate vectors $\mathbf{h}$ that lie within the ranges $0>h \geq-h_{\max }$; $k_{\max } \geq k \geq-k_{\max } ; l_{\max } \geq l \geq-l_{\max } ;$ and it was found that the limiting values of $h_{\max }=k_{\max }=l_{\max }=7$ were sufficient to cover the wavelength range of our measurements on SNAP. Note that only negative $h$ need be considered in our geometry since (as seen in Fig. 1) the reciprocal lattice points for all $h>0$ are outside of the Ewald volume and do not satisfy the Bragg condition.

The total transmission, $T^{\text {calc }}$, for the case of two diamonds is then the product of the two individual transmission spectra. This is scaled by a final scale factor $C$ to match the experimentally measured attenuation. In order that the final scaling retains $100 \%$ transmission where there is no dip, a constant of 1 is subtracted, the scale factor $C$ applied and then the constant 1 added back. This leads to the following final form for our empirical model for the total attenuation from both diamonds:

$$
T^{\mathrm{calc}}=C\left(T_{1}^{\mathrm{calc}} T_{2}^{\mathrm{calc}}-1\right)+1 .
$$


In order to compare this calculated dip spectrum with the measured spectrum, it is necessary first to transform the latter into a function of wavelength. The neutron wavelength is given by $\lambda_{\text {neut }}=h / p_{\text {neut }}$, where $h$ is Planck's constant and $p_{\text {neut }}$ the neutron momentum. As thermal neutrons are non-relativistic, their momentum is just the product of their mass $\left(m_{\text {neut }}\right)$ and velocity. The velocity is simply the distance $L$ from moderator to detector - which equals the sum of the distance from moderator to sample $\left(L_{1}\right)$ and sample to detector $\left(L_{2}\right)$ divided by TOF $(t)$. And, for an infinitesimally thin moderator, where $L$ is wavelength independent, $\lambda$ is exactly proportional to $t$. In reality, the moderator has a finite thickness, the effect of which can be modelled with an additional quadratic term, giving

$$
\lambda_{\text {neut }}=\left(\frac{h}{m_{\text {neut }}}\right) \frac{t}{L_{1}+L_{2}}+b t^{2} .
$$

Therefore, in addition to the model parameters described above, $L_{2}$ and $b$ are also refined in order to give the best fit between model and data.

In order to determine the best fitting parameters, the residual $\chi^{2}$ is defined as follows:

$$
\chi^{2}=\frac{1}{K} \sum_{1}^{K}\left(T^{\text {meas }}-T^{\text {calc }}\right)^{2} /\left(2 \delta T^{\text {meas } 2}\right),
$$

where $\delta T^{\text {meas }}$ are the standard deviations associated with the transmission measurement at each of the $K$ data points at

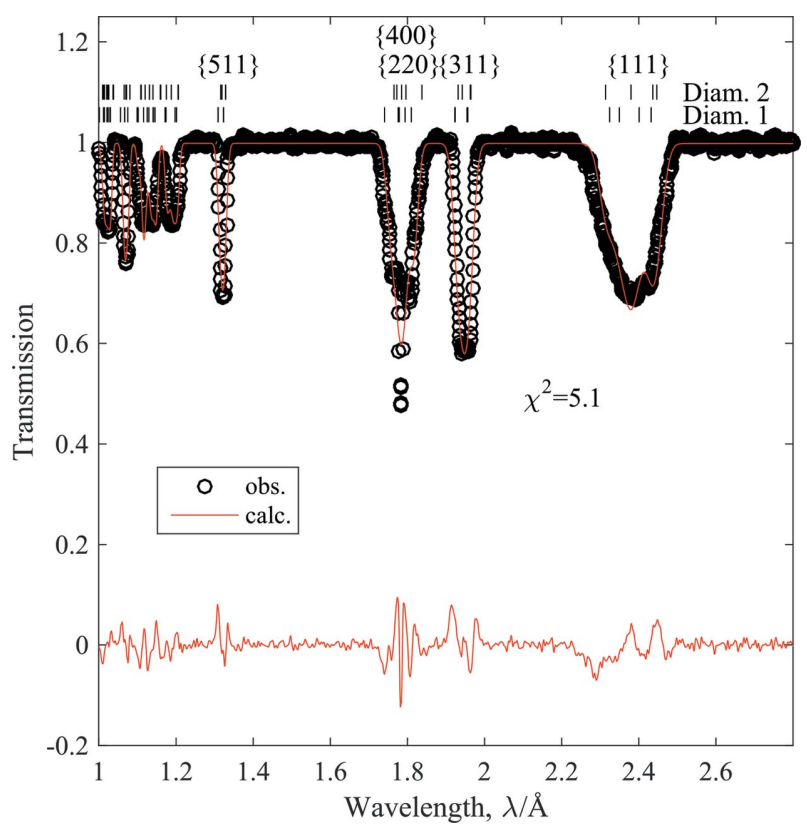

Figure 3

Fit to $\mathrm{Ni}$ transmission data measured at $0.8 \mathrm{GPa}$. The measured transmission data are shown as open circles as a function of wavelength. The solid red line running through the data points is the calculated dip pattern from the model described, and below the pattern the residual is also shown as a solid red line. Above the measured spectrum, the calculated positions of individual dips due to specific Bragg peaks are shown as vertical bars. The first few sets of dips are labelled with their $h k l$ class in curly braces. Low-wavelength dips are unlabelled to retain clarity. which is it measured. The best fit is then found by minimizing $\chi^{2}$ with respect to the variables of the model described above. In our case, minimization is achieved using the FMINCON routine in the MATLAB Optimization Toolbox (The MathWorks Inc., Natick, MA, USA), which finds the minimum of a general constrained nonlinear multivariable function.

This algorithm allows for the possibility of introducing constraints or restraints by defining upper and lower bounds for any of the variables in the fit. This utility is important at higher pressures where broadening increases the overlaps between adjacent peaks, increasing the likelihood of serial correlations between fitting parameters. As the peaks are sharpest in the lowest-pressure measurement, its refinement can often be run with less stringent constraints. The best fitting parameters obtained can then be used as initial conditions for the higher-pressure measurements and, where appropriate, restrained or fixed. One of the most important restraints is of the set of peak areas $\kappa_{n}$. The greatest stability is obtained by fixing these to be the same for each of the two diamonds and only refining a single scale factor between these.

Fig. 3 shows the result of applying the model to our lowestpressure Ni dataset at $0.8 \mathrm{GPa}$. The optimal adjustment angles $\alpha, \beta$ and $\gamma$ were found to be $0.1,-0.7$ and $-0.2^{\circ}$ for the upstream diamond and $0.0,-0.9$ and $-0.3^{\circ}$ for the downstream diamond. Although there are slight misfits related to peak shape and width that contribute to a high final $\chi^{2}$ of 5.0, the overall shape of the spectrum is well reproduced.

Once a fit to the full transmission dataset has been obtained, the attenuation due to each individual diamond can be extracted. These are shown in Fig. 4, where the different

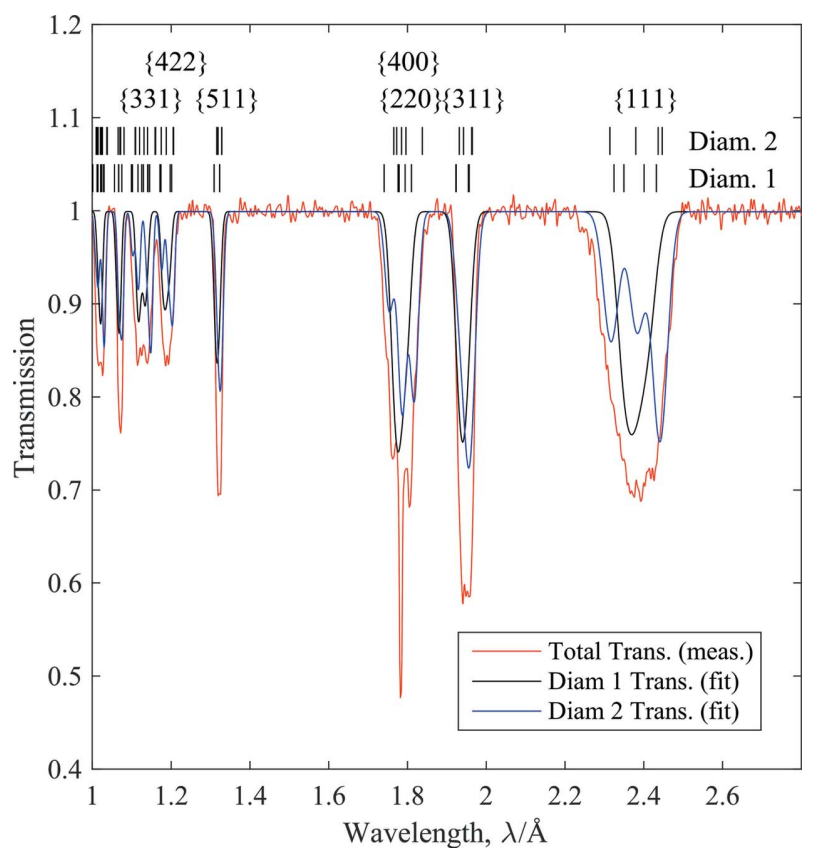

Figure 4

The individual contributions of each diamond, extracted by our empirical fitting approach, are shown with the total measured attenuation. Vertical bars above the pattern indicate the wavelength of individual dips. The first few sets of dips are labelled with their $h k l$ class. Low-wavelength dips are unlabelled to retain clarity. 
orientations of each diamond are seen to lead to distinct differences in the individual transmission spectra, notably in the total width of the dip clusters as seen, for example, in the four $\{111\}$-type dips between wavelengths of 2.25 and $2.50 \AA$ (note that we use curly braces to indicate the set of symmetryequivalent reflections within a dip).

We now consider the effect of the diamond attenuation on the sample Bragg reflection intensities. In TOF diffraction, a full diffraction pattern is measured in every detector pixel, the conversion between wavelength (determined from the measured TOF) and $d$ spacing being given by Bragg's law. The detectors on SNAP are highly pixelated and, in general, each pixel samples the diffraction pattern at a different scattering angle. Accordingly, the $i$ th pixel, with scattering angle $2 \theta_{i}$, will observe any given sample Bragg reflection, of $d$ spacing $d$, at a wavelength of $\lambda_{i}=2 d \sin \theta_{i}$. In order to maximize counting statistics, it is standard practice to combine the counts in all of the pixels within a detector bank. In this process, called 'time focusing', the data in each pixel are transformed from functions of TOF to functions of $d$ spacing, and then summed. This yields a total diffraction pattern, as a function of $d$ spacing, in which each Bragg reflection has been averaged over a finite wavelength range dictated by the distribution of angles $2 \theta_{i}$ that has been summed over. As the diamond attenuation affects only specific wavelengths, time-focused data will typically see a reduced effect due to diamond attenuation, with only a subset of pixels - those where the wavelength of a sample Bragg peak crosses that of a dip - being affected.

For diamond-anvil experiments, the distribution of pixels in which sample Bragg peaks are measured is complicated by the need for a software mask that excludes pixels containing

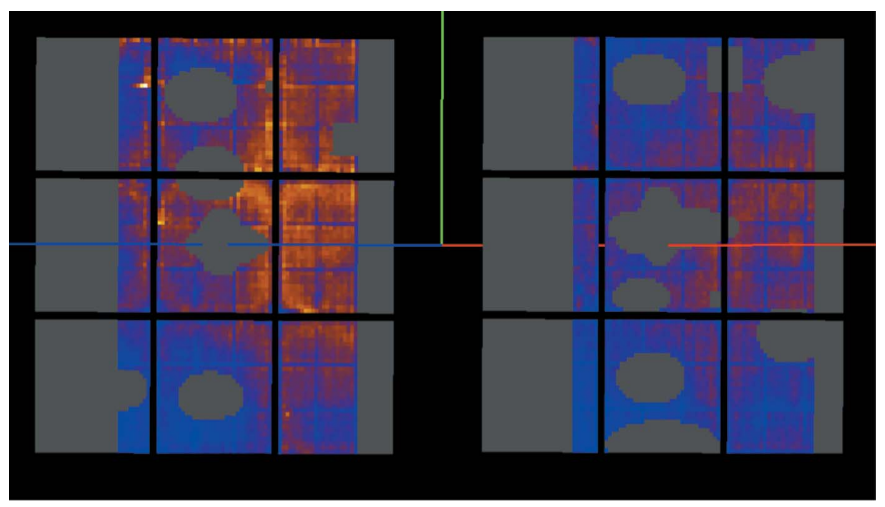

Figure 5

A two-dimensional plot of both detector panels on SNAP, showing in false colour the summed distribution of detected neutron intensity in the $d$-spacing range $0.65-3.1 \AA$ across both detector surfaces. The solid lines (blue, green and red) indicate the instrument frame, chosen such that the blue line (extending left from the origin) represents $\mathbf{x}$ (and is parallel to the incident beam) and the green line (extending vertically up from the origin) represents $\mathbf{z}$. Therefore, the left edge of each panel is at low $2 \theta$, while the right-hand edges are at high $2 \theta$. Both detectors are centred on $2 \theta=90^{\circ}$ and subtend a total angle of $\sim 45^{\circ}$. The areas shown in grey have been masked out and the coloured areas show detected neutron intensity in false colour, with blue indicating the lowest intensity and yellow/orange the highest. The black horizontal and vertical lines are gaps in angular coverage between the nine individual detector modules in each of the panels. strong anvil peaks and areas of the detector that are blocked by the cell body. During time focusing, all masked pixels are ignored and do not contribute to the final integrated diffraction pattern. The mask created for the $\mathrm{Ni}$ dataset is shown (coloured solid grey) in Fig. 5, where the vertical bars at the detector edges correspond to the diffraction aperture of the cell and the irregular shapes within the aperture correspond to diamond Bragg spots.

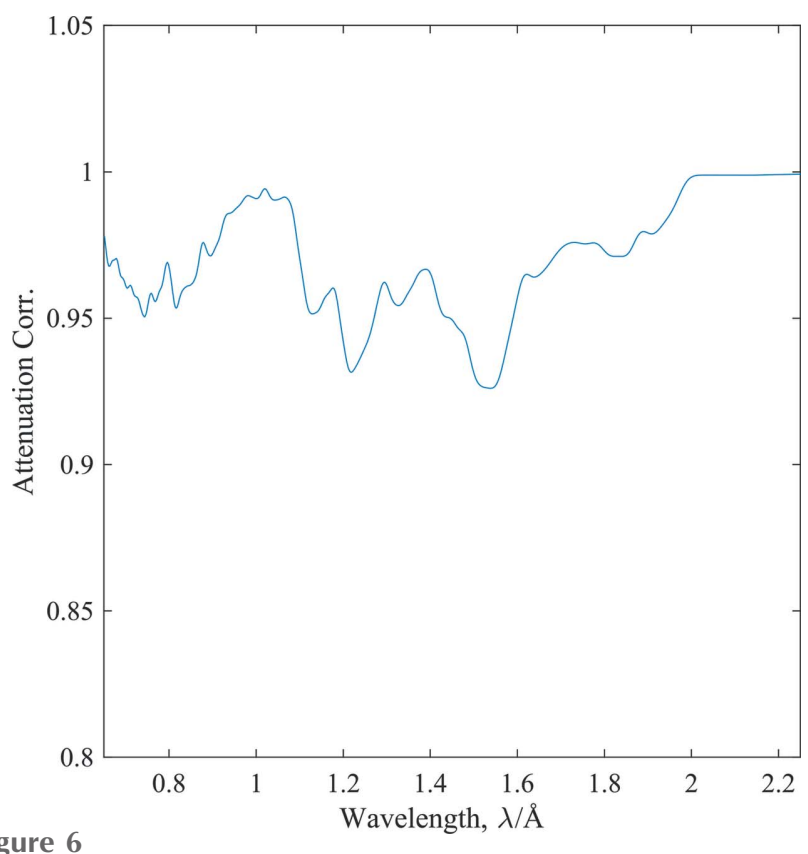

Figure 6

The time-focused total attenuation correction for our lowest-pressure data point $\sim 0.8 \mathrm{GPa}$. Under these conditions, attenuation is relatively weak, with a maximum attenuation of $\sim 7 \%$. This figure also illustrates that, for our geometry, the diamond attenuation has no effect on reflections measured above $d$ spacings of $2 \AA$.

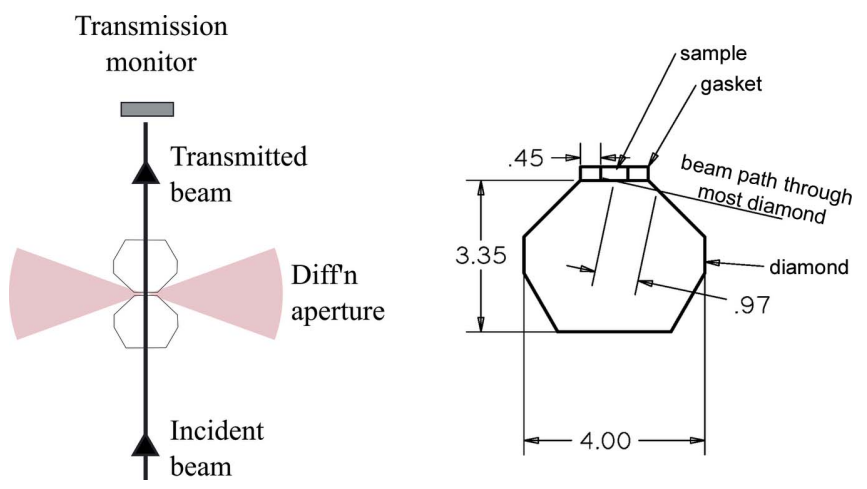

Figure 7

(Left) Schematic of diffraction geometry: the beam passes along the axis of the DAC, being attenuated by the upstream diamond before reaching the sample and then being attenuated by the downstream diamond before being measured in the transmission monitor. Diffraction from the sample and diamonds is measured within the indicated aperture, approximately $70<2 \theta<110^{\circ}$. (Right) Close-up of a single-crystal diamond anvil illustrating the longest beam path from sample to detector for a conventional scattering geometry. This path occurs at the largest scattering angle and for a small element of the sample closest to the diamond surface on the same side as the exiting beam path and furthest from the detector. Dimensions are given in millimetres. 
In order that the final attenuation function has the correct weighting of attenuation at each $d$ spacing, it is time focused using exactly the same mask as the data. The resulting total attenuation correction, now a function of $d$ spacing, is given in Fig. 6. Here, we see that the averaging affect of time focusing 'smears out' the diamond dip: broadening sharp features in wavelength space and reducing their depth. Correspondingly, the maximal effect of the dip on the diffraction pattern, seen at $\sim 1.5 \AA$ in $d$ spacing, is only $\sim 6 \%$ compared with an effect of $30 \%$ reduction in intensity transmitted by the upstream diamond at the worst affected wavelength of $\sim 2.3 \AA$ (see Fig. 4). Note also that there is no effect above a $d$ spacing of $\sim 2 \AA$, which corresponds to the $d$ spacing of the highestwavelength dip, measured at the lowest scattering angle.

The total attenuation function from the upstream diamond, $T_{1}^{\text {calc }}$, converted to $d$ spacing (Fig. 6) can be used directly to apply a correction to the (also time-focused) diffraction data, by simple division.

One final aspect of diamond attenuation that we considered is the possibility of effects on the diffracted as well as incident beams. Fig. 7 shows the geometry of a standard design of anvil currently used on SNAP. This immediately shows that the effect on the diffracted beam will be substantially smaller than that on the incident beam on account of the path length being $70 \%$ less. If we consider the total attenuation of the incident beam due to the $\{111\}$-type dips (shown in Fig. 4), which reduces the beam by $\sim 25 \%$, the maximum effect on the diffracted beam at the same wavelength would be only an $8 \%$ reduction.

\section{High-pressure data}

Once a framework to extract the upstream diamond attenuation and to generate a corresponding correction had been developed, we then proceeded to examine the effect of increased pressure on the transmission spectra. Table 1 shows the conditions of our measurements, while the measured transmission data themselves are shown in Fig. 8. A marked increase in the depth of dips is observed as sample pressure and mechanical strain on the diamonds increases, although, interestingly, some subsets of reflections, notably the $\{511\}$ type reflections (around $5200 \mu \mathrm{s}$ ) and the $\{620\}$-type reflections (around $4200 \mu \mathrm{s}$ ), appear to be less affected. This may reflect anisotropy in the mosaic spread of the diamond lattices.

In order to extract the attenuation correction for each pressure point, the analysis described above in $\$ 4$ was applied to each of the datasets in Fig. 8. The resulting attenuation, due to the upstream diamond only, is shown in Fig. 9. By the highest load, the dip due to the $\{111\}$-type collection of reflections (clustered between 2.25 and $2.5 \AA$ wavelength) is seen to absorb a full $75 \%$ of the incident beam.

If we consider the set of dips between 2.25 and $2.50 \AA$, which are due to the diamond $\{111\}$ set of reflections, the general trend of minimum transmission versus load can be extracted (Fig. 9). The transmission is seen to decrease approximately linearly as a function of load, reaching only $15 \%$ at the highest load. An increase of dip depth with anvil
Table 1

Experimental parameters for our Ni datasets.

Gas driving pressure was measured by an inline manometer and converted to force by means of an offline calibration that gave around $11 \pm 1$ bar per tonne ( 1 bar $=10^{5} \mathrm{~Pa}$ ). Sample pressures were determined from the refined unit-cell volumes using the equation of state of nickel reported by Dewaele et al. (2008).

\begin{tabular}{lll}
\hline Gas driving pressure (bar) & Force (tonnes) & Sample pressure $(\mathrm{GPa})$ \\
\hline 10 & 0.9 & 0.8 \\
15 & 1.3 & 1.2 \\
20 & 1.8 & 3.7 \\
25 & 2.2 & 9.8 \\
30 & 2.7 & 13.7 \\
35 & 3.2 & 17.2 \\
40 & 3.6 & 20.7 \\
45 & 4.0 & 24.1 \\
50 & 4.5 & 27.5 \\
55 & 5.0 & 30.9 \\
60 & 5.4 & 34.3 \\
65 & 5.8 & 37.5 \\
70 & 6.3 & 40.4 \\
\hline
\end{tabular}

strain was also observed by Loveday et al. (1990), who found that the intensity of the dips increased dramatically with pressure (e.g. the 111 class reflections were reflecting 20-30\% of the incident beam). However, in those measurements, the effect was mostly saturated by $0.2 \mathrm{GPa}$, at a force of not more than 0.012 tonnes $(1 \mathrm{t}=1000 \mathrm{~kg})$. This discrepancy may be due to substantial differences in the experimental setup, for
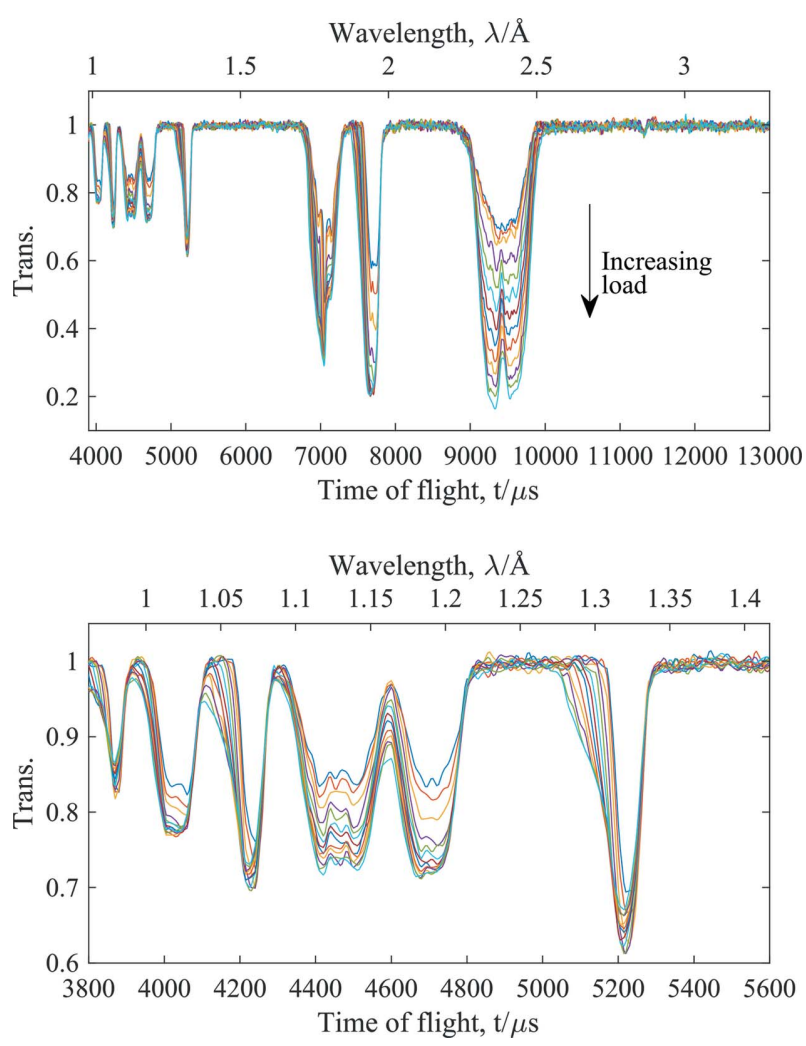

Figure 8

Total transmission spectra, as a function of TOF, with increasing force. The full measured spectra are shown in the upper panel, while the lower panel shows the low-TOF region. 
example source divergences and cell sizes. Also observed is broadening of the dips and shifting of position as the applied force strains the diamond crystal lattice.

The refined setting angles for both diamonds are shown in Fig. 10. These are seen to vary continuously with pressure. This might be due to slight mechanical deformation of the cell as the load increases.

As a final step, the extracted transmission of the upstream diamond is converted to $d$ spacing to provide the correction applicable to the sample diffraction data. This is shown in Fig. 11, which gives the final correction for each pressure measurement (as described previously, the same detector mask as used for focusing the sample diffraction patterns was used to generate the attenuation corrections). The correction is seen to have a relatively complex shape, with a maximum effect of around $25 \%$ at the highest load of 6.3 tonnes.

\section{Test of correction using diffraction detector}

Our fitting approach provides a means to decouple the attenuating effects of the two diamonds in order to extract the effect of only the upstream diamond. However, it is important to confirm that this procedure yields an accurate correction for the attenuation experienced by the sample. An independent means to examine this is to directly look for a wavelengthdependent dip in the intensity of Bragg peaks due to the sample as measured in the main diffraction detector banks. To do this, we examined our highest-pressure data set, where the attenuation effect is greatest, and looked at how Bragg intensity varies with angle (Fig. 12).

The longest and second longest
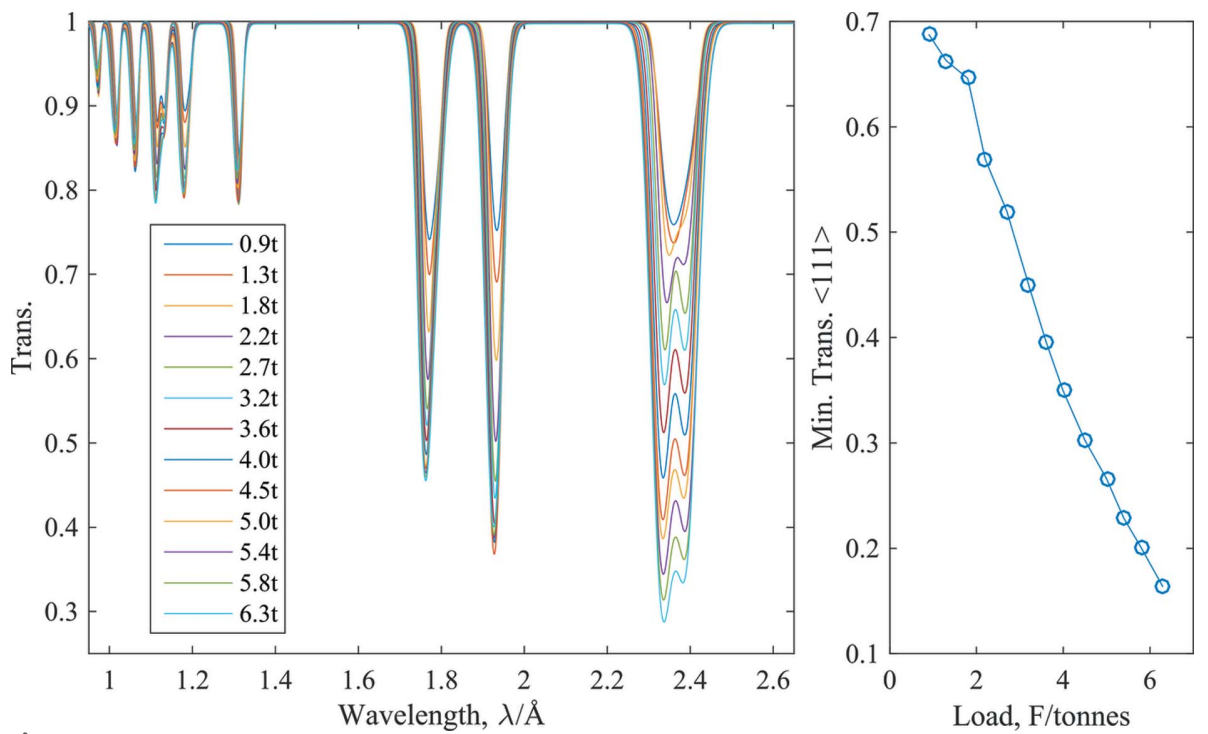

Figure 9

(Left) Fitted attenuation due to the upstream diamond only as a function of increasing load. As seen in the total measured pattern, the depth of dips increases systematically with pressure. (Right) The minimum transmission of the $\{111\}$ dip as a function of load.
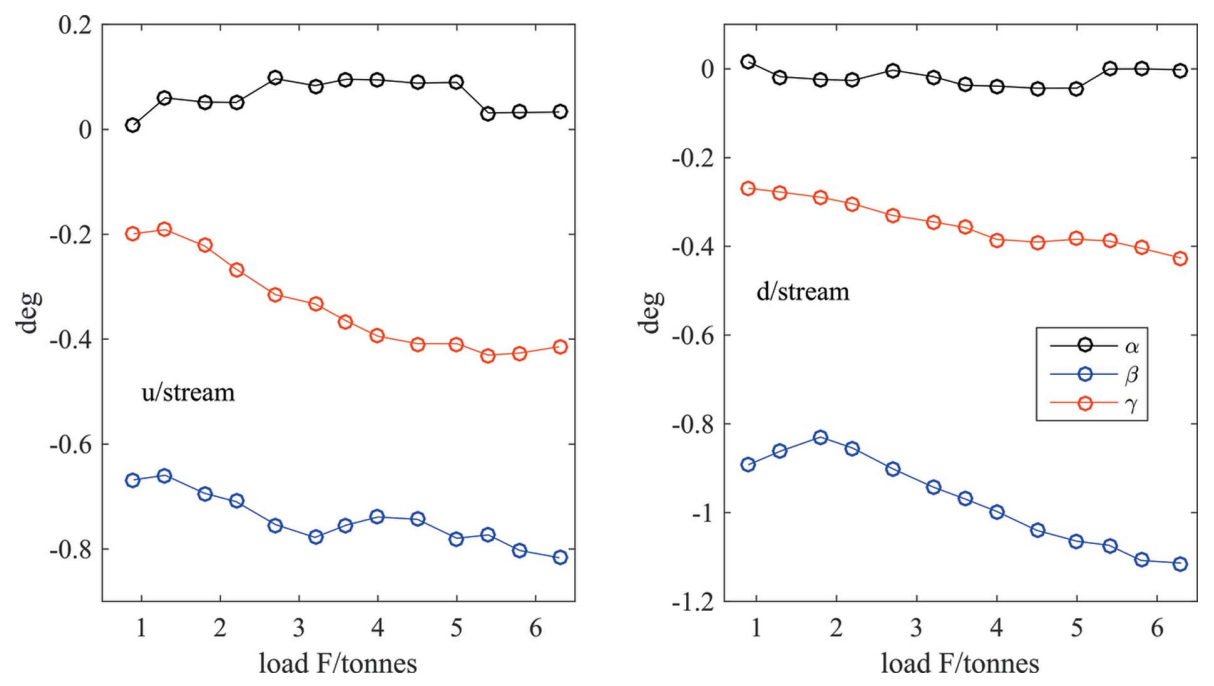

Figure 10

Adjustment angles for upstream (left) and downstream (right) diamonds as a function of load. $d$-spacing reflections from $\mathrm{Ni}$ are the 111 and 200 reflections, respectively, found at 1.93 and $1.67 \AA d$ spacing at this pressure. As seen in Fig. 12, both reflections transit through the $\mathrm{TOF} / \lambda$ of the dip with increasing angle. For all angles above $\sim 81^{\circ}, 111$ is completely unaffected by the dip and its intensity can be used to normalize that of 200 as it enters, then exits, the dip. By fitting Gaussian peak shapes to the individual TOF spectra at each scattering angle, we extracted the ratio of intensities, to look for direct evidence of a reduction and then recovery of the ratio as 200 enters and then exits the dip. As shown in Fig. 13, we see that the ratio does indeed drop as 200 enters the dip and recover again as it exits the dip on the other side. The apparent greater width of the dip observed in the diffraction detectors is attributed to a miscalibration of the relative angular position of the detector modules.

\section{Rietveld analysis of attenuation correction}

Our reference sample of $\mathrm{Ni}$ powder was chosen because it is a strong scatterer and has Bragg peaks at appropriate $d$ spacings to sample the attenuation. In addition, it retains its face-centred-cubic structure across our full pressure range so that the relative peak intensities should be constant (apart from systematic effects due to changes in thermal motion). To confirm this, each of the datasets listed in 


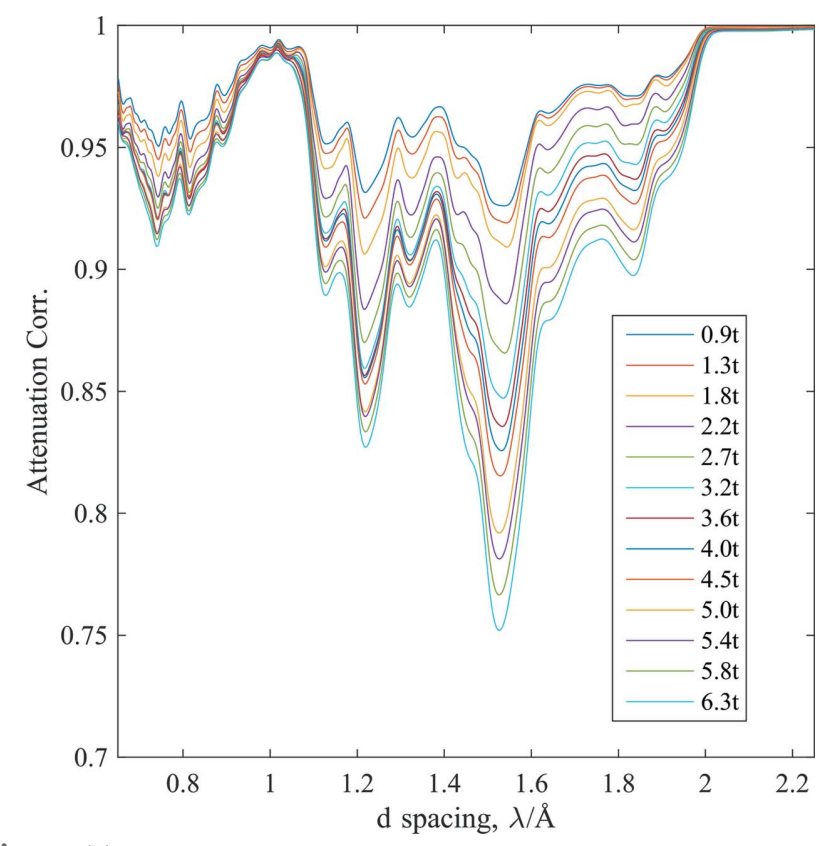

Figure 11

Final upstream diamond attenuation correction, after conversion to $d$ spacing, as a function of increasing load.

Table 1 was subject to a Rietveld refinement to examine the efficacy of the correction.

Prior to sample data collection, a $500 \mu \mathrm{m}$ steel pin was placed at the sample position and a dataset measured. Bragg peaks from the known structure of the steel were used to calibrate the $\mathrm{TOF}-\lambda$ conversion for the pixels in the detector. Subsequently, a $610 \mu \mathrm{m}$-diameter vanadium pin and a separate background measurement were taken to correct for various systematic $\lambda$-dependent effects, including incident spectrum profile, detector efficiency etc. This yielded a $\mathrm{V}$ correction function that could be applied to the sample data to normalize for these effects.

For each $\mathrm{Ni}$ dataset, a mask was applied to remove the single-crystal diamond peaks from the background. ${ }^{1}$ After application of the mask, the data were focused as described in $\S 4$ to give a diffraction pattern as a function of $d$ spacing. Subsequently, a smooth $d$-dependent background function was subtracted from the Ni datasets, which were then divided by the $\mathrm{V}$ correction function. A correction for the attenuation due to the steel gasket (Guthrie, Boehler, Molaison et al., 2013) was calculated but was found to be negligible. In addition, no attenuation correction was applied to the $\mathrm{V}$ measurement, owing to the small diameter of the pin (calculations indicate that the effect is $\sim 2 \%$ across the entire $d$-spacing range that was refined). The resulting datasets are fully corrected for all instrumental effects other than the diamond attenuation. These were refined to give a reference and, subsequently, the diamond attenuation correction described above was applied and the data re-refined. The

\footnotetext{
${ }^{1}$ The same mask used for the Ni sample was also applied to the corrected $\mathrm{V}$ data sets in order to correctly account for the angular distribution of unmasked pixels and differing efficiency of individual detector pixels.
}

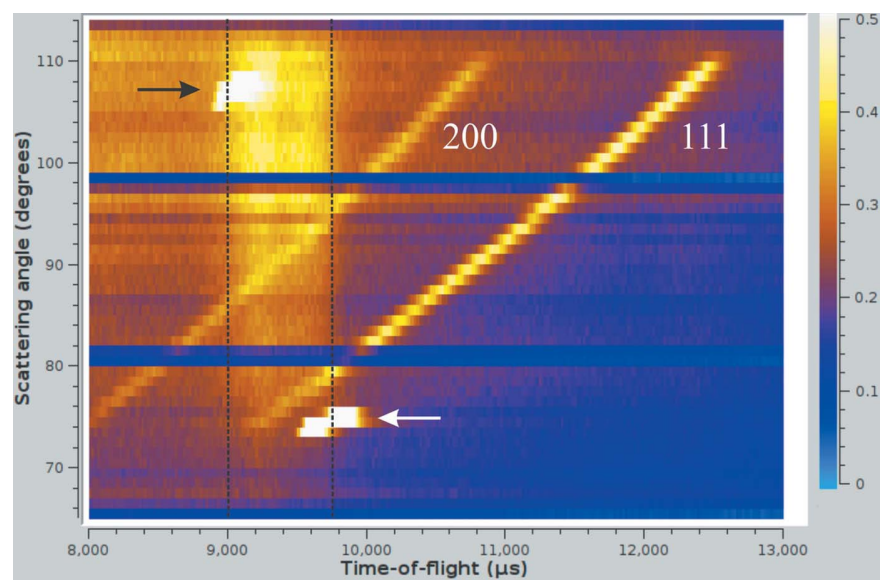

Figure 12

False colour image generated by the Mantidplot software package (Taylor et al., 2012), showing the scattered intensity as a function of $2 \theta$ versus TOF, measured in the $90^{\circ}$ SNAP diffraction banks. The 111 and $200 \mathrm{Ni}$ diffraction peaks sweep across the image at an oblique angle. The TOF regions affected by Bragg dips are clearly visible as the radiation lost in transmission is redistributed as additional background (probably due to secondary $\gamma$-radiation). Correspondingly, the dip due to the $\{111\}$ diamond reflections is seen as a positive band of intensity running vertically up the image, with the outline marked by vertical dashed lines (note that the TOF of this region differs from that of the transmission data as the diffraction detectors have a different secondary path length compared to the transmission monitor). The saturated spots indicated by arrows are single-crystal diamond diffraction spots. The dark horizontal bands at 81 and $98^{\circ}$ are due to gaps in angular coverage between individual detector modules (see also Fig. 5). The loss of sample Bragg intensity at the highest and lowest angles is due to the limited angular window of the cell.

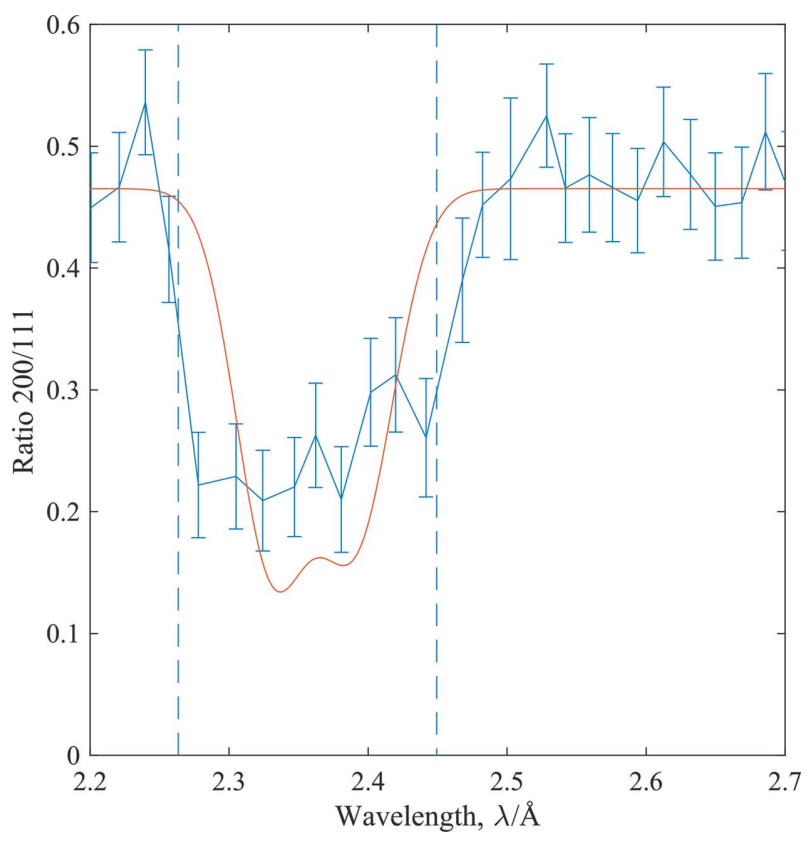

Figure 13

The ratio of intensities of the 200 and 111 Bragg reflections from Fig. 12, with the $x$ axis converted from TOF to wavelength to compare with the calculated attenuation. Dashed vertical lines indicate where 200 enters and exits the dip. The continuous red line is the calculated attenuation scaled to the 200/111 ratio at wavelengths unaffected by the dip. 
difference between the two refinements encapsulates the effect of the diamond dips.

All datasets were refined using the GSAS (Larson \& Von Dreele, 1994) and EXPGUI (Toby, 2001) Reitveld software packages. The known structure of nickel (space group: Fm3m with an $\mathrm{Ni}$ atom at $0,0,0)$ was used, for which the only refinable structural parameters were the lattice parameter $a$ and an isotropic atomic displacement parameter for the $\mathrm{Ni}$ atom. In addition to these structural parameters, both Gaussian and Lorentzian peak shape and three background parameters were required to describe the pattern. During refinements, a small amount of preferred orientation was observed, which increased with pressure. This was fitted with a spherical harmonic model that introduced a single additional parameter. In total this gave nine parameters in the fit. The fitting range was defined so that all diffraction patterns had the same number of observed reflections, which were all of those including and above the $\{422\}$ class of reflections. Pressure was determined from the refined lattice parameter using the Deweale et al. (2008) equation of state.

First, we compared the quality of fit, measured in the refinement by the integrated weighted residual $\chi^{2}$, which is shown in Fig. 14.

While the diamond attenuation has very little effect at the lowest pressures, it increases rapidly with pressure and, consequently, we see a substantial improvement in fit after the attenuation correction is applied. In addition, the trend of $\chi^{2}$ with pressure appears to show more scatter without the attenuation correction, as the refinement attempts to fit the affected reflections using other available parameters. This is
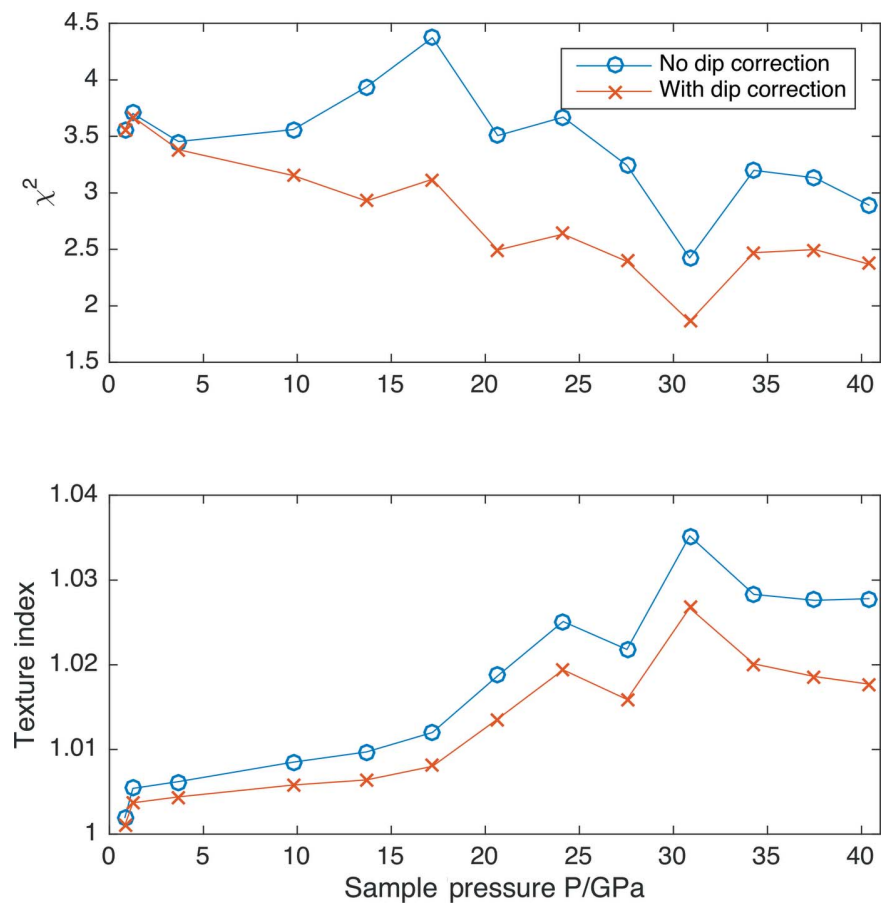

Figure 14

(Upper panel) The $\chi^{2}$ values obtained in refinements for both uncorrected and corrected datasets. (Lower panel) The texture index (Toby, 2001) for both refinements. illustrated in the refined texture index, which quantifies the preferred orientation. Although the amount of preferred orientation is quite small, it is systematically larger without the attenuation correction, leading to an overestimate of the texture (Fig. 14).

\section{Discussion}

The effect of applied load on the depth and width of the dips may have a number of origins. For example, significant primary extinction is expected, which returns radiation from the diffracted to the incident beam with a phase shift of $\pi$, cancelling some part of the radiation as it travels through the diamond. Applying load will induce strain gradients in the diamonds which will reduce such extinction and increase the intensity of the beam diffracted by the diamond and hence the depth of the dip. Strain gradients in the diamond will also increase the acceptance angle of the diamond, determined by measuring a rocking curve, which increases the proportion of the incident fan of radiation that the diamond diffracts. A precise model of how the depth of the dip varies with load is beyond the scope of this paper - since we are using the measured transmission - but it would be of interest to understand why the depth of some classes of dip are more strongly load dependent.

\section{Summary}

In summary, we have developed a methodology to correct polychromatic TOF diffraction data measured inside diamond anvil cells for the effect of diamond attenuation. This semiempirical approach uses measurement of total transmitted intensities recorded by a neutron monitor that is downstream of the sample cell. These data are then fitted with a model, allowing the separate extraction of the upstream diamond contribution. We have validated the resulting correction by direct measurement of the attenuation effect on Bragg intensities as they scan across the diffraction detectors.

We also explored the effect of the final attenuation correction on the measurement of Bragg intensities from a reference sample of $\mathrm{Ni}$ compressed to $\sim 40 \mathrm{GPa}$. As the $\mathrm{Ni}$ structure is known, the quality of fit is a measure of how effectively the effect of diamond dips is mitigated by applying the correction. We found substantial improvements of up to $25 \%$ in the $\chi^{2}$ value of the fit after applying the dip correction, indicating that, after correction, reliable Bragg intensities can be extracted.

\section{Acknowledgements}

We thank Jon Taylor for assistance in implementing the MantidPlot software and Richard Nelmes for useful discussions. The internal-membrane diamond anvil cell used in this work was developed with the support of the Deep Carbon Observatory. CGP is supported by a studentship from the Scottish Doctoral Training Centre in Condensed Matter 
Physics, which is funded in part by the UK Engineering and Physical Sciences Research Council (EPSRC). MED is supported by an EPSRC doctoral training programme studentship. Part of MG's contribution to this work was supported by EFree, an Energy Frontier Research Center funded by the US Department of Energy (DOE), Office of Science, Office of Basic Energy Sciences (BES), under award DE-SC0001057.

\section{References}

Angel, R. J. (2004). J. Appl. Cryst. 37, 486-492.

Ashcroft, N. W. \& Mermin, N. D. (1976). Solid State Physics. New York: Holt

Chatterjee, A., Mikkelson, D., Mikkelson, R., Hammonds, J. \& Worlton, T. (2002). Appl. Phys. Mater. Sci. Process. 74, s194s197.

Dewaele, A., Torrent, M., Loubeyre, P. \& Mezouar, M. (2008). Phys. Rev. B, 78, 104102.
Giacovazzo, C. (1998). Editor. Fundamentals of Crystallography. Oxford University Press.

Guthrie, M., Boehler, R., Molaison, J. J., dos Santos, A. M. \& Tulk, C. A. (2013). Am. Crystallogr. Assoc. Trans. Ser. 44, 149-169.

Guthrie, M., Boehler, R., Tulk, C. A., Molaison, J. J., dos Santos, A. M., Li, K. \& Hemley, R. J. (2013). Proc. Natl Acad. Sci. USA, 110, 10552-10556.

Larson, A. C. \& Von Dreele, R. B. (1994). Report LAUR 86-748. Los Alamos National Laboratory, New Mexico, USA.

Le Bail, A. (2005). Powder Diffr. 20, 316-326.

Loveday, J. S., McMahon, M. I. \& Nelmes, R. J. (1990). J. Appl. Cryst. 23, 392-396.

Richards, J. D., Cooper, R. G., Donahue, N. \& Visscher, T. (2010). IEEE Nuclear Science Symposium and Medical Imaging Conference, pp. 1771-1776. IEEE.

Sato, T., Ohashi, K., Sudoh, T., Haruna, K. \& Maeta, H. (2002). Phys. Rev. B, 65, 092102.

Sears, V. (1992). Neutron News, 3(3), 26-37.

Taylor, J., Arnold, O., Bilheaux, J., Buts, A., Campbell, S., Doucet, M., Draper, N., Fowler, R. \& Gigg, M. (2012). Bull. Am. Phys. Soc. 57, http://meetings.aps.org/link/BAPS.2012.MAR.W26.10.

Toby, B. H. (2001). J. Appl. Cryst. 34, 210-213. 